\title{
Statistical Convergence of Double Sequences in Locally Solid Riesz Spaces
}

\author{
S. A. Mohiuddine, ${ }^{1}$ Abdullah Alotaibi, ${ }^{1}$ and M. Mursaleen ${ }^{2}$ \\ ${ }^{1}$ Department of Mathematics, Faculty of Science, King Abdulaziz University, \\ P.O. Box 80203, Jeddah 21589, Saudi Arabia \\ ${ }^{2}$ Department of Mathematics, Aligarh Muslim University, Aligarh 202002, India
}

Correspondence should be addressed to S. A. Mohiuddine, mohiuddine@gmail.com

Received 26 May 2012; Accepted 13 August 2012

Academic Editor: Ziemowit Popowicz

Copyright (C) 2012 S. A. Mohiuddine et al. This is an open access article distributed under the Creative Commons Attribution License, which permits unrestricted use, distribution, and reproduction in any medium, provided the original work is properly cited.

Recently, the notion of statistical convergence is studied in a locally solid Riesz space by Albayrak and Pehlivan (2012). In this paper, we define and study statistical $\tau$-convergence, statistical $\tau$ Cauchy and $S^{*}(\tau)$-convergence of double sequences in a locally solid Riesz space.

\section{Introduction and Preliminaries}

The notion of statistical convergence was introduced by Fast [1] and Steinhaus [2] independently in the same year 1951. Actually, Henry Fast had heard about this concept from Steinhaus, but in fact it was Antoni Zygmund who proved theorems on the statistical convergence of Fourier series in the first edition of his book ([3], pp. 181-188) where he used the term "almost convergence" in place of statistical convergence and at that time this idea was not recognized much. Since the term "almost convergence" was already in use (see Lorentz [4]), Fast had to choose a different name for his concept and "statistical convergence" was mostly the suitable one. Active researches on this topic started after the papers of Fridy [5] and since then a huge amount of literature has appeared. It developed so rapidly like an explosion when the summability theory was at the dying stage. Various extensions, generalizations, variants, and applications have been given by several authors so far, for example [6-16]. This notion has also been defined and studied in different setups, for example in a locally convex space [17]; in topological groups [18, 19]; in probabilistic normed spaces [20]; in intuitionistic fuzzy normed spaces [21]; in fuzzy/random 2-normed space [22, 23]. Recently, Albayrak and Pehlivan [24] studied this notion in locally solid Riesz spaces. In this paper, we study statistically convergent, statistically bounded, and statistically Cauchy double sequences in locally solid Riesz spaces. 
Let $X$ be a real vector space and $\leq$ a partial order on this space. Then, $X$ is said to be an ordered vector space if it satisfies the following properties:

(i) if $x, y \in X$ and $y \leq x$, then $y+z \leq x+z$ for each $z \in X$.

(ii) if $x, y \in X$ and $y \leq x$, then $\lambda y \leq \lambda x$ for each $\lambda \geq 0$.

If in addition $X$ is a lattice with respect to the partially order $\leq$, then $X$ is said to be a Riesz space (or a vector lattice) [25].

For an element $x$ of a Riesz space $X$, the positive part of $x$ is defined by $x^{+}=x \vee \theta=$ $\sup \{x, \theta\}$, the negative part of $x$ by $x^{-}=(-x) \vee \theta$ and the absolute value of $x$ by $|x|=x \vee(-x)$, where $\theta$ is the zero element of $X$.

A subset $S$ of a Riesz space $X$ is said to be solid if $y \in S$ and $|x| \leq|y|$ implies $x \in S$.

A topological vector space $(X, \tau)$ is a vector space $X$ which has a (linear) topology $\tau$ such that the algebraic operations of addition and scalar multiplication in $X$ are continuous. Continuity of addition means that the function $f: X \times X \rightarrow X$ defined by $f(x, y)=x+y$ is continuous on $X \times X$, and continuity of scalar multiplication means that the function $f$ : $\mathbb{C} \times X \rightarrow X$ defined by $f(\lambda, x)=\lambda x$ is continuous on $\mathbb{C} \times X$.

Every linear topology $\tau$ on a vector space $X$ has a base $\mathcal{N}$ for the neighborhoods of $\theta$ satisfying the following properties:

$\left(C_{1}\right)$ each $Y \in \mathcal{N}$ is a balanced set, that is, $\lambda x \in Y$ holds for all $x \in Y$ and every $\lambda \in \mathbb{R}$ with $|\lambda| \leq 1$.

$\left(C_{2}\right)$ each $Y \in \mathcal{N}$ is an absorbing set, that is, for every $x \in X$, there exists $\lambda>0$ such that $\lambda x \in Y$.

$\left(C_{3}\right)$ for each $Y \in \mathcal{N}$ there exists some $E \in \mathcal{N}$ with $E+E \subseteq Y$.

A linear topology $\tau$ on a Riesz space $X$ is said to be locally solid [26] if $\tau$ has a base at zero consisting of solid sets. A locally solid Riesz space $(X, \tau)$ is a Riesz space equipped with a locally solid topology $\tau$.

By the convergence of a double sequence we mean the convergence in the Pringsheim's sense [27]. A double sequence $x=\left(x_{j k}\right)$ is said to converge to the limit L in Pringsheim's sense (shortly, $P$-convergent to $L$ ) if for every $\varepsilon>0$ there exists an integer $N$ such that $\left|x_{j k}-L\right|<\varepsilon$ whenever $j, k>N$. In this case $L$ is called the $P$-limit of $x$.

A double sequence $x=\left(x_{j k}\right)$ of real or complex numbers is said to be bounded if $\|x\|_{\infty}=$ $\sup _{j, k}\left|x_{j k}\right|<\infty$. The space of all bounded double sequences is denoted by $\boldsymbol{M}_{u}$.

\section{Statistical $\tau$-Convergence}

Let $K \subseteq \mathbb{N} \times \mathbb{N}$ be a two-dimensional set of positive integers and let $K(m, n)=\{(j, k): j \leq$ $m, k \leq n\}$. Then the two-dimensional analogue of natural density can be defined as follows.

In case the sequence $(K(m, n) / m n)$ has a limit in Pringsheim's sense, then we say that $K$ has a double natural density and is defined as

$$
P-\lim _{m, n} \frac{K(m, n)}{m n}=\delta_{2}(K) .
$$


For example, let $K=\left\{\left(i^{2}, j^{2}\right): i, j \in \mathbb{N}\right\}$. Then,

$$
\delta_{2}(K)=P-\lim \frac{K(m, n)}{m n} \leq P-\lim _{m, n} \frac{\sqrt{m} \sqrt{n}}{m n}=0,
$$

That is, the set $K$ has double natural density zero, while the set $\{(i, 2 j): i, j \in \mathbb{N}\}$ has double natural density $1 / 2$.

A real double sequence $x=\left(x_{j k}\right)$ is said to be statistically convergent (see [28-30]) to the number $\ell$ if for each $\epsilon>0$, the set

$$
\left\{(j, k), j \leq m, k \leq n:\left|x_{j k}-\ell\right| \geq \epsilon\right\}
$$

has double natural density zero.

We shall assume throughout this paper that the symbol $\mathcal{N}_{\text {sol }}$ will denote any base at zero consisting of solid sets and satisfying the conditions $\left(C_{1}\right),\left(C_{2}\right)$, and $\left(C_{3}\right)$ in a locally solid topology.

Definition 2.1. Let $(X, \tau)$ be a locally solid Riesz space. Then, a double sequence $x=\left(x_{j k}\right)$ in $X$ is said to be statistically $\tau$-convergent to the number $\xi \in X$ if for every $\tau$-neighborhood $U$ of zero:

$$
P-\lim _{m, n \rightarrow \infty} \frac{1}{m n}\left|\left\{(j, k), j \leq m, k \leq n: x_{j k}-\xi \notin U\right\}\right|=0
$$

In this case, we write $\mathcal{S}(\tau)-\lim x=\xi$ or $x_{j k} \stackrel{\mathcal{S}(\tau)}{\longrightarrow} \xi$.

Definition 2.2. Let $(X, \tau)$ be a locally solid Riesz space. We say that a double sequence $x=\left(x_{j k}\right)$ in $X$ is statistically $\tau$-bounded if for every $\tau$-neighborhood $U$ of zero there exists some $\lambda>0$, such that the set

$$
\left\{(j, k), j \leq m, k \leq n: \lambda x_{j k} \notin U\right\}
$$

has double natural density zero.

Theorem 2.3. Let $(X, \tau)$ be a Hausdorff locally solid Riesz space and $x=\left(x_{j k}\right)$ and $y=\left(y_{j k}\right)$ be two double sequences in $X$. Then, the following hold:

(i) if $\mathcal{S}(\tau)-\lim _{j, k} x_{j k}=\xi_{1}$ and $\mathcal{S}(\tau)-\lim _{j, k} x_{j k}=\xi_{2}$, then $\xi_{1}=\xi_{2}$.

(ii) if $\mathcal{S}(\tau)-\lim _{j, k} x_{j k}=\xi$, then $\mathcal{S}(\tau)-\lim _{j, k} \alpha x_{j k}=\alpha \xi, \alpha \in \mathbb{R}$.

(iii) if $\mathcal{S}(\tau)-\lim _{j, k} x_{j k}=\xi$ and $\mathcal{S}(\tau)-\lim _{j, k} y_{j k}=\eta$, then $\mathcal{S}(\tau)-\lim _{j, k}\left(x_{j k}+y_{j k}\right)=\xi+\eta$.

Proof. (i) Suppose that $\mathcal{S}(\tau)-\lim _{j, k} x_{j k}=\xi_{1}$ and $\mathcal{S}(\tau)-\lim _{j, k} x_{j k}=\xi_{2}$. Let $U$ be any $\tau$-neighborhood of zero. Then, there exists $Y \in \mathcal{N}_{\text {sol }}$ such that $Y \subseteq U$. Choose any $E \in \mathcal{N}_{\text {sol }}$ such that $E+E \subseteq Y$. We define the following sets:

$$
\begin{aligned}
& K_{1}=\left\{(j, k), j \leq m, k \leq n: x_{j k}-\xi_{1} \in E\right\}, \\
& K_{2}=\left\{(j, k), j \leq m, k \leq n: x_{j k}-\xi_{2} \in E\right\} .
\end{aligned}
$$


Since $\mathcal{S}(\tau)-\lim _{j, k} x_{j k}=\xi_{1}$ and $\mathcal{S}(\tau)-\lim _{j, k} x_{j k}=\xi_{2}$, we have $\delta_{2}\left(K_{1}\right)=\delta_{2}\left(K_{2}\right)=1$. Thus, $\delta_{2}\left(K_{1} \cap\right.$ $\left.K_{2}\right)=1$, and in particular $K_{1} \cap K_{2} \neq \emptyset$. Now, let $(j, k) \in K_{1} \cap K_{2}$. Then

$$
\xi_{1}-\xi_{2}=\xi_{1}-x_{j k}+x_{j k}-\xi_{2} \in E+E \subseteq Y \subseteq U
$$

Hence for every $\tau$-neighborhood $U$ of zero, we have $\xi_{1}-\xi_{2} \in U$. Since $(X, \tau)$ is Hausdorff, the intersection of all $\tau$-neighborhoods $U$ of zero is the singleton set $\{\theta\}$. Thus, we get $\xi_{1}-\xi_{2}=\theta$, that is, $\xi_{1}=\xi_{2}$.

(ii) Let $U$ be an arbitrary $\tau$-neighborhood of zero and $\mathcal{S}(\tau)-\lim _{j, k} x_{j k}=\xi$. Then there exists $Y \in \mathcal{N}_{\text {sol }}$ such that $Y \subseteq U$ and also

$$
P-\lim _{m, n \rightarrow \infty} \frac{1}{m n}\left|\left\{(j, k), j \leq m, k \leq n: x_{j k}-\xi \in Y\right\}\right|=1 .
$$

Since $Y$ is balanced, $x_{j k}-\xi \in Y$ implies that $\alpha\left(x_{j k}-\xi\right) \in Y$ for every $\alpha \in \mathbb{R}$ with $|\alpha| \leq 1$. Hence,

$$
\begin{aligned}
\left\{(j, k), j \leq m, k \leq n: x_{j k}-\xi \in Y\right\} & \subseteq\left\{(j, k), j \leq m, k \leq n: \alpha x_{j k}-\alpha \xi \in Y\right\} \\
& \subseteq\left\{(j, k), j \leq m, k \leq n: \alpha x_{j k}-\alpha \xi \in U\right\} .
\end{aligned}
$$

Thus, we obtain

$$
P-\lim _{m, n \rightarrow \infty} \frac{1}{m n}\left|\left\{(j, k), j \leq m, k \leq n: \alpha x_{j k}-\alpha \xi \in U\right\}\right|=1
$$

for each $\tau$-neighborhood $U$ of zero. Now let $|\alpha|>1$ and $[|\alpha|]$ be the smallest integer greater than or equal to $|\alpha|$. There exists $E \in \mathcal{N}_{\text {sol }}$ such that $[|\alpha|] E \subseteq Y$. Since $\mathcal{S}(\tau)-\lim _{j, k} x_{j k}=\xi$, the set

$$
K=\left\{(j, k), j \leq m, k \leq n: x_{j k}-\xi \in E\right\}
$$

has double natural density zero. Therefore,

$$
\left|\alpha \xi-\alpha x_{j k}\right|=|\alpha|\left|\xi-x_{j k}\right| \leq[|\alpha|]\left|\xi-x_{j k}\right| \in[|\alpha|] E \subseteq Y \subseteq U
$$

Since the set $Y$ is solid, we have $\alpha \xi-\alpha x_{j k} \in Y$. This implies that $\alpha \xi-\alpha x_{j k} \in U$. Thus,

$$
P-\lim _{m, n \rightarrow \infty} \frac{1}{m n}\left|\left\{(j, k), j \leq m, k \leq n: \alpha x_{j k}-\alpha \xi \in U\right\}\right|=1,
$$

for each $\tau$-neighborhood $U$ of zero. Hence, $S(\tau)-\lim _{j, k} \alpha x_{j k}=\alpha \xi$. 
(iii) Let $U$ be an arbitrary $\tau$-neighborhood of zero. Then there exists $Y \in \mathcal{N}_{\text {sol }}$ such that $Y \subseteq U$. Choose $E$ in $\mathcal{N}_{\text {sol }}$ such that $E+E \subseteq Y$. Since $\mathcal{S}(\tau)-\lim _{j, k} x_{j k}=\xi$ and $\mathcal{S}(\tau)-\lim _{j, k} y_{j k}=\eta$, we have $\delta_{2}\left(H_{1}\right)=1=\delta_{2}\left(H_{2}\right)$, where

$$
\begin{aligned}
& H_{1}=\left\{(j, k), j \leq m, k \leq n: x_{j k}-\xi \in E\right\}, \\
& H_{2}=\left\{(j, k), j \leq m, k \leq n: y_{j k}-\eta \in E\right\} .
\end{aligned}
$$

Let $H=H_{1} \cap H_{2}$. Hence, we have $\delta_{2}(H)=1$ and

$$
\left(x_{j k}+y_{j k}\right)-(\xi+y)=\left(x_{j k}-x\right)+\left(y_{j k}-\eta\right) \in E+E \subseteq Y \subseteq U \text {. }
$$

Therefore,

$$
P-\lim _{m, n \rightarrow \infty} \frac{1}{m n}\left|\left\{(j, k), j \leq m, k \leq n:\left(x_{j k}+y_{j k}\right)-(\xi+\eta) \in U\right\}\right|=1 .
$$

Since $U$ is arbitrary, we have $\mathcal{S}(\tau)-\lim _{j, k}\left(x_{j k}+y_{j k}\right)=\xi+\eta$.

This completes the proof of the theorem.

Theorem 2.4. Let $(X, \tau)$ be a locally solid Riesz space. If a double sequence $x=\left(x_{j k}\right)$ is statistically $\tau$-convergent, then it is statistically $\tau$-bounded.

Proof. Suppose $x=\left(x_{j k}\right)$ is statistically $\tau$-convergent to the point $\xi \in X$ and let $U$ be an arbitrary $\tau$-neighborhood of zero. Then, there exists $Y \in \mathcal{N}_{\text {sol }}$ such that $Y \subseteq U$. Let us choose $E \in \mathcal{N}_{\text {sol }}$ such that $E+E \subseteq Y$. Since $\mathcal{S}(\tau)-\lim _{j, k \rightarrow \infty} x_{j k}=\xi$, the set

$$
K=\left\{(j, k), j \leq m, k \leq n: x_{j k}-\xi \notin E\right\}
$$

has double natural density zero. Since $E$ is absorbing, there exists $\lambda>0$ such that $\lambda \xi \in E$. Let $\alpha$ be such that $\alpha \leq 1$ and $\alpha \leq \lambda$. Since $E$ is solid and $|\alpha \xi| \leq|\lambda \xi|$, we have $\alpha \xi \in E$. Since $E$ is balanced, $x_{j k}-\xi \in E$ implies that $\alpha\left(x_{j k}-\xi\right) \in E$. Then, we have

$$
\alpha x_{j k}=\alpha\left(x_{j k}-\xi\right)+\alpha \xi \in E+E \subseteq Y \subseteq U,
$$

for each $(j, k) \in \mathbb{N} \times \mathbb{N} \backslash K$. Thus

$$
P-\lim _{m, n \rightarrow \infty} \frac{1}{m n}\left|\left\{(j, k), j \leq m, k \leq n: \alpha x_{j k} \notin U\right\}\right|=0 .
$$

Hence, $\left(x_{j k}\right)$ is statistically $\tau$-bounded.

This completes the proof of the theorem. 
Theorem 2.5. Let $(X, \tau)$ be a locally solid Riesz space. If $\left(x_{j k}\right),\left(y_{j k}\right)$, and $\left(z_{j k}\right)$ are three double sequences such that

(i) $x_{j k} \leq y_{j k} \leq z_{j k}$ for all $j, k \in \mathbb{N}$;

(ii) $\mathcal{S}(\tau)-\lim _{j, k} x_{j k}=\xi=\mathcal{S}(\tau)-\lim _{j, k} z_{j k}$;

then $\mathcal{S}(\tau)-\lim _{j, k} y_{j k}=\xi$.

Proof. Let $U$ be an arbitrary $\tau$-neighborhood of zero, there exists $Y \in \mathcal{N}_{\text {sol }}$ such that $Y \subseteq U$. Choose $E \in \mathcal{N}_{\text {sol }}$ such that $E+E \subseteq Y$. From condition (ii), we have $\delta_{2}(A)=1=\delta_{2}(B)$, where

$$
\begin{aligned}
& A=\left\{(j, k), j \leq m, k \leq n: x_{j k}-\xi \in E\right\} \\
& B=\left\{(j, k), j \leq m, k \leq n: z_{j k}-\xi \in E\right\} .
\end{aligned}
$$

Now, let $Q=A \cap B$. Then, from (i), we have

$$
x_{j k}-\xi \leq y_{j k}-\xi \leq z_{j k}-\xi,
$$

this implies

$$
\left|y_{j k}-\xi\right| \leq\left|x_{j k}-\xi\right|+\left|z_{j k}-\xi\right| \in E+E \subseteq Y
$$

for each $(j, k) \in Q$. Since $Y$ is solid, we have $y_{j k}-\xi \in Y \subseteq U$. Thus,

$$
P-\lim _{m, n \rightarrow \infty} \frac{1}{m n}\left|\left\{(j, k), j \leq m, k \leq n: y_{j k}-\xi \in U\right\}\right|=1,
$$

for each $\tau$-neighborhood $U$ of zero. Hence $\mathcal{S}(\tau)-\lim _{j, k} y_{j k}=\xi$.

This completes the proof of the theorem.

\section{Statistically $\tau$-Cauchy and $\mathcal{S}^{*}(\tau)$-Convergence}

Definition 3.1. Let $(X, \tau)$ be a locally solid Riesz space. A double sequence $x=\left(x_{j k}\right)$ in $X$ is statistically $\tau$-Cauchy if for every $\tau$-neighborhood $U$ of zero there exist $N, M \in \mathbb{N}$ such that for all $j, p \geq N, k, q \geq M$, the set

$$
\left\{(j, k), j \leq m, k \leq n: x_{j k}-x_{p q} \notin U\right\}
$$

has double natural density zero.

Theorem 3.2. Let $(X, \tau)$ be a locally solid Riesz space. If a double sequence $x=\left(x_{j k}\right)$ is statistically $\tau$-convergent, then it is statistically $\tau$-Cauchy.

Proof. Suppose that $\mathcal{S}(\tau)-\lim _{j, k \rightarrow \infty} x_{j k}=\xi$. Let $U$ be an arbitrary $\tau$-neighborhood of zero, there exists $Y \in \mathcal{N}_{\text {sol }}$ such that $Y \subseteq U$. Choose $E \in \mathcal{N}_{\text {sol }}$ such that $E+E \subseteq Y$. Then,

$$
P-\lim _{m, n \rightarrow \infty} \frac{1}{m n}\left|\left\{(j, k), j \leq m, k \leq n: x_{j k}-\xi \notin E\right\}\right|=0 .
$$


Also, we have

$$
x_{j k}-x_{p q}=x_{j k}-\xi+\xi-x_{p q} \in E+E \subseteq Y \subseteq U,
$$

for all $(j, k),(p, q) \in \mathbb{N} \times \mathbb{N} \backslash K$, where

$$
K=\left\{(j, k), j \leq m, k \leq n: x_{j k}-\xi \notin E\right\} .
$$

Therefore, the set

$$
\left\{(j, k), j \leq m, k \leq n: x_{j k}-x_{p q} \notin U\right\} \subseteq K
$$

For every $\tau$-neighborhood $U$ of zero there exist $N, M \in \mathbb{N}$ such that for all $j, p \geq N, k, q \geq M$, the set $\left\{(j, k), j \leq m\right.$ and $\left.k \leq n: x_{j k}-x_{p q} \notin U\right\}$ has double natural density zero. Hence, $\left(x_{j k}\right)$ is statistically $\tau$-Cauchy.

This completes the proof of the theorem.

In [30], it was shown that a real double sequence $x=\left(x_{j k}\right)$ is statistically convergent to a number $\ell$ if and only if there exists a subset $K=\{(j, k)\} \subseteq \mathbb{N} \times \mathbb{N}, j, k=1,2, \ldots$ such that $\delta_{2}(K)=1$ and $\lim _{j, k \rightarrow \infty} x_{j k}=\ell$.

This fact suggests defining further another type of convergence in locally solid Riesz spaces.

Definition 3.3. A sequence $\left(x_{j k}\right)$ in a locally solid Riesz space $(X, \tau)$ is said to $\mathcal{S}^{*}(\tau)$-convergent to $\xi \in X$ if there exists a set $K=\{(j, k)\} \subseteq \mathbb{N} \times \mathbb{N}, j, k=1,2, \ldots$, with $\delta_{2}(K)=1$ such that $\lim _{j, k \rightarrow \infty} x_{j k}=\xi$. In this case, we write $\xi=\mathcal{S}^{*}(\tau)$-lim $x$.

Theorem 3.4. A double sequence $x=\left(x_{j k}\right)$ is statistically $\tau$-convergent to a number $\xi$ if it is $S^{*}(\tau)$ convergent to $\xi$ in a locally solid Riesz space $(X, \tau)$.

Proof. Let $U$ be an arbitrary $\tau$-neighborhood of $\xi$. Since $x=\left(x_{j k}\right)$ is $\mathcal{S}^{*}(\tau)$-convergent to $\xi$, there is a set $K=\{(j, k)\} \subseteq \mathbb{N} \times \mathbb{N}, j, k=1,2, \ldots$, with $\delta_{2}(K)=1$ and $j_{0}=j_{0}(U), k_{0}=k_{0}(U)$ such that $j \geq j_{0}, k \geq k_{0}$, and $(j, k) \in K$ imply that $x_{j k}-\xi \in U$. Then,

$$
K_{U}=\left\{(j, k) \in \mathbb{N} \times \mathbb{N}: x_{j k}-\xi \notin U\right\} \subseteq \mathbb{N} \times \mathbb{N}-\left\{\left(j_{N+1}, k_{N+1}\right),\left(j_{N+2}, k_{N+2}\right), \ldots\right\}
$$

Therefore,

$$
\delta_{2}\left(K_{U}\right) \leq 1-1=0 .
$$

Hence, $x$ is statistically $\tau$-convergent to $\xi$.

This completes the proof of the theorem.

Note that the converse holds for first countable space. 
Recall that a first countable space is a topological space satisfying the "first axiom of countability." Specifically, a space $X$ is said to be first countable if each point has a countable neighbourhood basis (local base), that is, for each point $x$ in $X$ there exists a sequence $U_{1}, U_{2}, \ldots$ of open neighbourhoods of $x$ such that for any open neighbourhood $V$ of $x$ there exists an integer $i$ with $U_{i}$ contained in $V$.

Theorem 3.5. Let $(X, \tau)$ be a first countable locally solid Riesz space. If a double sequence $x=\left(x_{j k}\right)$ is statistically $\tau$-convergent to a number $\xi$, then it is $\mathcal{S}^{*}(\tau)$-convergent to $\xi$.

Proof. Let $x$ be statistically $\tau$-convergent to a number $\xi$. Fix a countable local base $U_{1} \supset U_{2} \supset$ $U_{3} \supset \cdots$ at $\xi$. For every $i \in \mathbb{N}$, put

$$
\begin{gathered}
K_{i}=\left\{(j, k) \in \mathbb{N} \times \mathbb{N}: x_{j k}-\xi \notin U_{i}\right\}, \\
M_{i}=\left\{(j, k) \in \mathbb{N} \times \mathbb{N}: x_{j k}-\xi \in U_{i}\right\} \quad(r=1,2,3, \ldots) .
\end{gathered}
$$

Then, $\delta_{2}\left(K_{i}\right)=0$ and

(1) $M_{1} \supset M_{2} \supset \cdots \supset M_{i} \supset M_{i+1} \supset \cdots$;

(2) $\delta_{2}\left(M_{i}\right)=1, i=1,2,3, \ldots$

Now we have to show that for $(j, k) \in M_{i},\left(x_{j k}\right)$ is convergent to $\xi$. Suppose that $\left(x_{j k}\right)$ is not convergent to $\xi$. Therefore, $x_{j k} \notin U_{i}$ for infinitely many terms. Let

$$
M_{r}=\left\{(j, k) \in \mathbb{N} \times \mathbb{N}: x_{j k}-\xi \in U_{r}\right\} \quad(r>i)
$$

Then,

(3) $\delta_{2}\left(M_{r}\right)=0$,

and by (1), $M_{i} \subset M_{r}$. Hence, $\delta_{2}\left(M_{i}\right)=0$ which contradicts (2). Therefore, $\left(x_{j k}\right)$ is convergent to $\xi$. Hence by Definition 3.3, $x$ is $\mathcal{S}^{*}(\tau)$-convergent to $\xi$.

This completes the proof of the theorem.

\section{References}

[1] H. Fast, "Sur la convergence statistique," Colloquium Mathematicum, vol. 2, pp. 241-244, 1951.

[2] H. Steinhaus, "Sur la convergence ordinaire et la convergence asymptotique," Colloquium Mathematicum, vol. 2, pp. 73-34, 1951.

[3] A. Zygmund, Trigonometric Series, Cambridge University Press, New York, NY, USA, 1st edition, 1959.

[4] G. G. Lorentz, "A contribution to the theory of divergent sequences," Acta Mathematica, vol. 80, pp. 167-190, 1948.

[5] J. A. Fridy, “On statistical convergence," Analysis, vol. 5, no. 4, pp. 301-313, 1985.

[6] R. Çolak and Ç. A. Bektaş, " $\lambda$-statistical convergence of order $\alpha$," Acta Mathematica Scientia Series B, vol. 31, no. 3, pp. 953-959, 2011.

[7] V. Kumar and M. Mursaleen, "On $(\lambda, \mu)$-statistical convergence of double sequences on intuitionistic fuzzy normed spaces," Filomat, vol. 25, no. 2, pp. 109-120, 2011.

[8] S. A. Mohiuddine and Q. M. D. Lohani, "On generalized statistical convergence in intuitionistic fuzzy normed space," Chaos, Solitons \& Fractals, vol. 42, no. 3, pp. 1731-1737, 2009.

[9] S. A. Mohiuddine and M. Aiyub, "Lacunary statistical convergence in random 2-normed spaces," Applied Mathematics E Information Sciences, vol. 6, no. 3, pp. 581-585, 2012. 
[10] M. Mursaleen and A. Alotaibi, "On I-convergence in random 2-normed spaces," Mathematica Slovaca, vol. 61, no. 6, pp. 933-940, 2011.

[11] S. A. Mohuiddine, A. Alotaibi, and S. M. Alsulami, "Ideal convergence of double sequences in 2 random 2-normed spaces," Advances in Difference Equations, vol. 2012, article 149, 2012.

[12] M. Mursaleen and S. A. Mohiuddine, "On lacunary statistical convergence with respect to the intuitionistic fuzzy normed space," Journal of Computational and Applied Mathematics, vol. 233, no. 2, pp. $142-149,2009$.

[13] M. Mursaleen and S. A. Mohiuddine, "On ideal convergence of double sequences in probabilistic normed spaces," Mathematical Reports, vol. 12, no. 4, pp. 359-371, 2010.

[14] M. Mursaleen and S. A. Mohiuddine, "On ideal convergence in probabilistic normed spaces," Mathematica Slovaca, vol. 62, no. 1, pp. 49-62, 2012.

[15] M. Mursaleen, S. A. Mohiuddine, and O. H. H. Edely, "On the ideal convergence of double sequences in intuitionistic fuzzy normed spaces," Computers $\mathcal{E}$ Mathematics with Applications, vol. 59, no. 2, pp. 603-611, 2010.

[16] E. Savaş and S. A. Mohiuddine, " $\bar{\lambda}$-statistically convergent double sequences in probabilistic normed spaces," Mathematica Slovaca, vol. 62, no. 1, pp. 99-108, 2012.

[17] I. J. Maddox, "Statistical convergence in a locally convex space," Mathematical Proceedings of the Cambridge Philosophical Society, vol. 104, no. 1, pp. 141-145, 1988.

[18] H. Cakalli, "On statistical convergence in topological groups," Pure and Applied Mathematika Sciences, vol. 43, no. 1-2, pp. 27-31, 1996.

[19] H. Çakalli and E. Savaş, "Statistical convergence of double sequences in topological groups," Journal of Computational Analysis and Applications, vol. 12, no. 2, pp. 421-426, 2010.

[20] S. A. Mohiuddine and E. Savaş, "Lacunary statistically convergent double sequences in probabilistic normed spaces," Annali dell'Universita di Ferrara. In press.

[21] M. Mursaleen and S. A. Mohiuddine, "Statistical convergence of double sequences in intuitionistic fuzzy normed spaces," Chaos, Solitons E Fractals, vol. 41, no. 5, pp. 2414-2421, 2009.

[22] S. A. Mohiuddine, H. Şevli, and M. Cancan, "Statistical convergence in fuzzy 2-normed space," Journal of Computational Analysis and Applications, vol. 12, no. 4, pp. 787-798, 2010.

[23] M. Mursaleen, "On statistical convergence in random 2-normed spaces," Acta Scientiarum Mathematicarum, vol. 76, no. 1-2, pp. 101-109, 2010.

[24] H. Albayrak and S. Pehlivan, "Statistical convergence and statistical continuity on locally solid Riesz spaces," Topology and its Applications, vol. 159, no. 7, pp. 1887-1893, 2012.

[25] A. C. Zaanen, Introduction to Operator Theory in Riesz Spaces, Springer, Berlin, Germany, 1997.

[26] G. T. Roberts, "Topologies in vector lattices," Mathematical Proceedings of the Cambridge Philosophical Society, vol. 48, pp. 533-546, 1952.

[27] A. Pringsheim, "Zur Theorie der zweifach unendlichen Zahlenfolgen," Mathematische Annalen, vol. 53, no. 3, pp. 289-321, 1900.

[28] M. Mursaleen, C. Çakan, S. A. Mohiuddine, and E. Savaş, "Generalized statistical convergence and statistical core of double sequences," Acta Mathematica Sinica, vol. 26, no. 11, pp. 2131-2144, 2010.

[29] M. Mursaleen and O. H. H. Edely, "Generalized statistical convergence," Information Sciences, vol. 162, no. 3-4, pp. 287-294, 2004.

[30] Mursaleen and O. H. H. Edely, "Statistical convergence of double sequences," Journal of Mathematical Analysis and Applications, vol. 288, no. 1, pp. 223-231, 2003. 


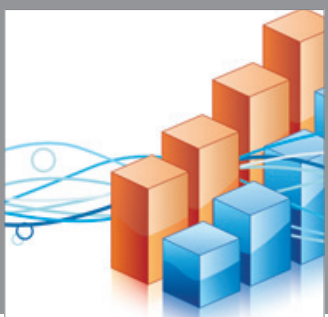

Advances in

Operations Research

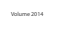

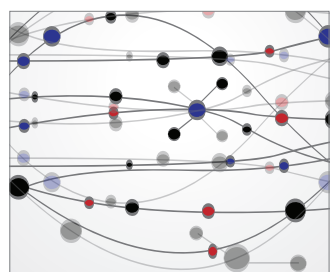

\section{The Scientific} World Journal
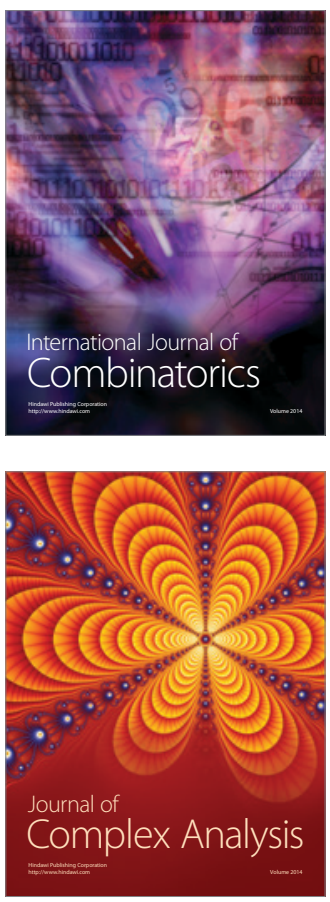

International Journal of

Mathematics and

Mathematical

Sciences
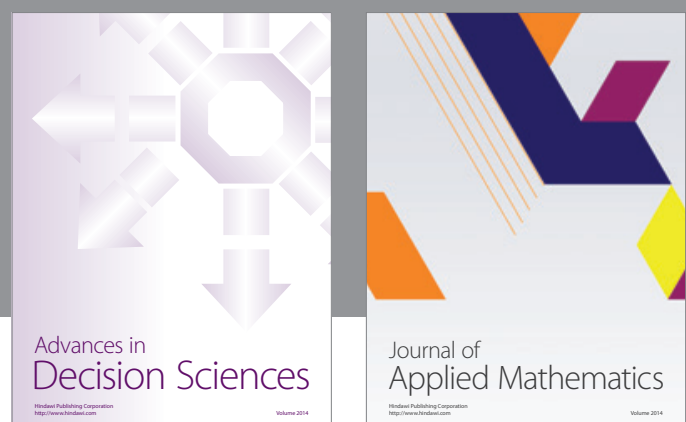

Journal of

Applied Mathematics
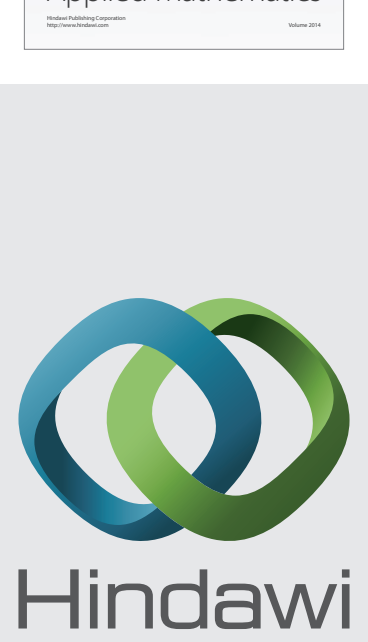

Submit your manuscripts at http://www.hindawi.com
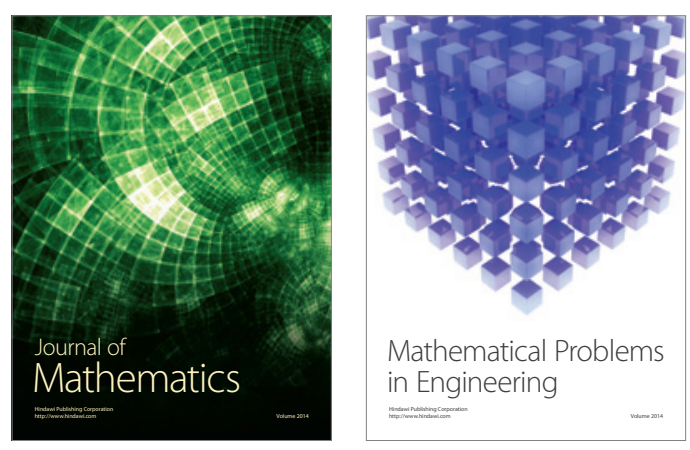

Mathematical Problems in Engineering
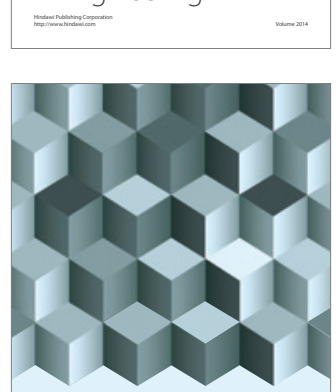

Journal of

Function Spaces
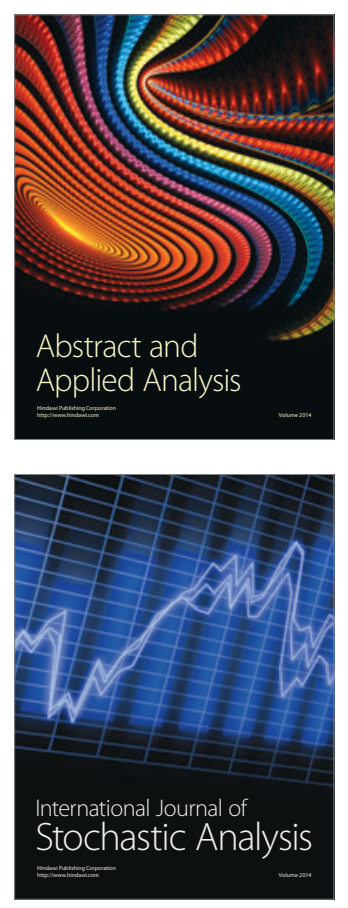

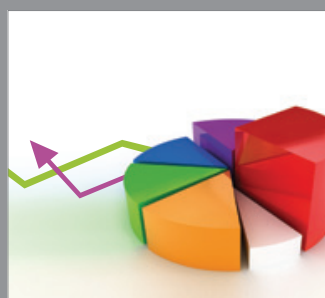

ournal of

Probability and Statistics

Promensencen
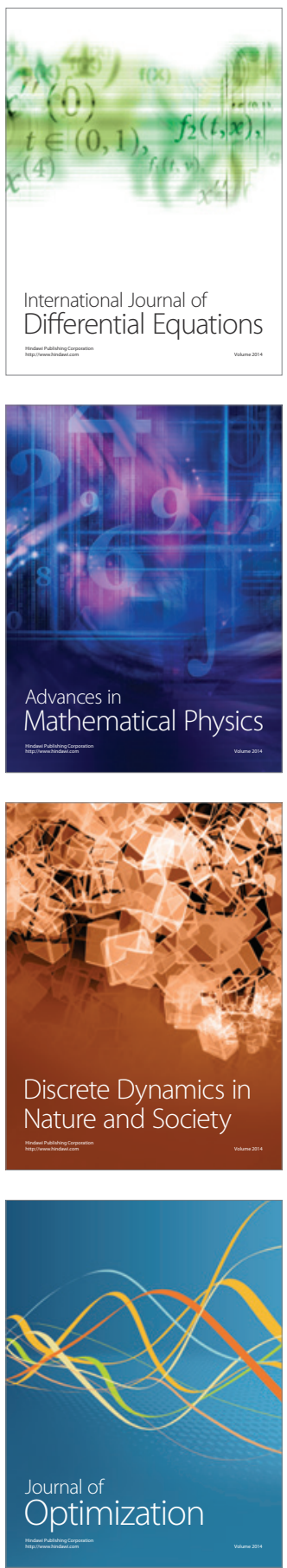